\title{
Activation of Akt (protein kinase B) stimulates metaphase I to metaphase II transition in bovine oocytes
}

\author{
Wolfgang Tomek and Tatjana Smiljakovic ${ }^{1}$ \\ Unit of Reproductive Biology, FBN Research Institute for the Biology of Farm Animals, Wilhelm-Stahl-Allee 2, \\ 18196 Dummerstorf, Germany and ${ }^{1}$ Institute of Animal Husbandry, Belgrade, Serbia
}

Correspondence should be addressed to W Tomek; Email: tomek@fbn-dummerstorf.de

\begin{abstract}
In somatic cells, the serine/threonine kinase Akt (or protein kinase B) was shown to contribute to processes linked to cellular growth, cell survival and cell cycle regulation. In contrast to these findings, the function of Akt during the meiosis of mammalian oocytes remains to be investigated. We analysed the phosphorylation pattern and the activity of Akt during meiotic maturation (transition from prophase I to metaphase II) of bovine oocytes. The oocytes were matured in vitro (IVM) for 0,10 and $24 \mathrm{~h}$ to reach the germinal vesicle (GV), metaphase I (M I) and metaphase II (M II) stages respectively. The abundance and phosphorylation pattern of Akt was revealed by Western blotting using total Akt or phosphoso-Akt-specific antibodies. The activity of this particular kinase was determined by an in vitro kinase assay. Furthermore, functional properties were analysed by cultivating oocytes in the presence of the Akt inhibitor SH6. The results showed that the overall abundance of Akt did not change significantly during IVM. On the other hand, Akt became phosphorylated at Thr 308 and Ser 473, reaching its maximum at the M I phase. In the GV and M II stages, only low basal phosphorylation levels were observed on both sides. This phosphorylation profile corresponded strictly to the activity of the kinase. The cultivation of oocytes in the presence of the phosphatidylinositol analogue $\mathrm{SH} 6$ for $24 \mathrm{~h}$ showed that, with higher concentrations, up to $65 \%$ of the oocytes were arrested in the M I stage. This result indicated that Akt is involved in the M I/M II transition during the meiotic maturation of bovine oocytes. The physiological aspects of the Akt function will be discussed.

Reproduction (2005) 130 423-430
\end{abstract}

\section{Introduction}

Mammalian oocytes are arrested in their follicles at the diplotene (dictyate) stage of the first meiotic prophase, the so-called germinal vesicle (GV) stage. When fully grown oocytes are released from their follicles, they can resume meiosis spontaneously if cultured in a suitable culture medium. This process (transition from the GV stage to metaphase II (M II)) is referred to as meiotic maturation of oocytes. During meiotic maturation, characteristic morphological changes occur. These include condensation of the chromatin, germinal vesicle breakdown (GVBD) and formation of the meiotic spindle.

It is generally known that the processes of cellular proliferation, differentiation or maturation are triggered by signalling cascades involving the action of protein kinases which specifically phosphorylate distinct targets. In this context, phosphorylation activates or deactivates proteins which are responsible for the progression of the cell cycle and, especially in oocytes, for the resumption of meiotic maturation from the arrested GV stage (Chian et al. 2003, Kishmoto 2003).
Several years ago, a factor was postulated to affect the onset of the meiotic maturation of oocytes. This factor was named maturation-promoting factor (MPF). With the discovery of cyclins (Evans et al. 1983, Gerhard et al. 1984, for review see Dorée \& Hunt 2002) it became evident that the MPF is a cyclin-dependent kinase (CDK) and consists of a catalytic subunit Cdc2 (cell division cycle kinase) and a regulatory subunit cyclin B (Jessus \& Beach 1992, Claude et al. 2002, Jones 2004). The MPF is thought to be a universal G2/M-phase regulator for both meiosis and mitosis (Ye et al. 2003) in all eukaryotic cells (the GV stage of meiosis is usually assumed to be equivalent to the G2/M border in mitosis (Moor et al. 1990)). An evaluation of the activation profiles of distinct kinases during the transition from the GV to the M II stage in oocytes revealed that several kinases are present during this period, but not all of them are in an activated state. These kinases include, besides the MPF, the proto-oncogene c-Mos (which is an MAP kinase (MAPK) kinase), MAPK-extracellular activating kinase (MEK), which is a substrate of c-Mos and phosphorylates and activates MAPK) and MAPK itself (Nebreda \& 
Hunt 1993, Shibuya \& Ruderman 1993). The activity of Cdc2 kinase, which is said to initiate events leading to nuclear progression, is high during GVBD and M II (Tian et al. 2002), low after the MI phase, and the kinase is inactive during the GV stage. MAPK, on the other hand, which was shown to be involved in translational regulation in bovine oocytes by its stimulation of phosphoryation of the initiation factor elF4E (eucaryotic initiation factor $4 \mathrm{E}$, which binds to the $5^{\prime}$-cap structure of mRNA; Tomek et al. 2002a) is inactive only in the GV stage, after which it becomes phosphorylated; its activity steadily increases, reaching its maximum in $\mathrm{M}$ II. The upstream kinase MEK also shows an activity pattern corresponding to that of MAPK (Liu \& Yang 1999).

It was previously shown that another kinase named Akt (protein kinase B; PKB) is also abundant in mouse and bovine oocytes (Hoshino et al. 2004, Vigneron et al. 2004a, 2004b). The results reported indicate that Akt is likely to participate in the maturation of oocytes, probably independent of MPF activation.

In somatic cells, Akt was shown to contribute to networks integrating growth, survival and proliferation of cells (Massague 2004). In this context, Akt is involved, for instance, in CDK activation, inhibition of caspase activation and the stimulation of translation. In contrast to these observations taken from somatic cells, much less is known about the molecular function of Akt during oocyte maturation. Recently published results obtained from starfish oocytes indicate that Akt stimulates the meiotic G2/M-phase transition by down-regulating the activity of Myt 1 (Okumura et al. 2002). In this way, the inhibitory phosphorylation of the MPF is impaired and this factor can be activated by cdc25 phosphatase. These observations identify Akt as an M-phase initiator, but do not necessarily reflect the situation in mammalian oocytes.

The activation of Akt depends on the phosphorylation at Thr 308 and Ser 473. It was shown previously that the Thr 308 residue is phosphorylated by 3-phosphoinositidedependent protein kinase-1 (PDK1) and that membrane localization is a necessary criterion for Ser 473 phosphorylation (Scheid et al. 2002, Troussard et al. 2003). New results have shown that in Drosophila and human somatic cells the target of rapamycin kinase and its associated protein rictor are necessary for the phosphorylation at Ser 473 (Sarbassov et al. 2005). Furthermore, results obtained from lung cancer cell lines indicate that phosphatidylinositol analogues can prevent Ser 473 phosphorylation and specifically impair Akt activation. In this context it is of interest that SH6 was reported to block Akt activation without affecting either the upstream kinase PDK1 or other kinases downstream of Ras, such as MAPK (Kozikowski et al. 2003).

With regard to the facts described above, we investigated the phosphorylation pattern of Akt during in vitro maturation (IVM) of bovine oocytes. Furthermore, we elucidated the activity of this kinase, using the novel phosphatidylinositol analogue SH6 to investigate the influence of Akt inhibition on the meiotic maturation of bovine oocytes. This approach can contribute to the acquisition of more detailed information on molecular events during the meiotic maturation of oocytes.

\section{Materials and Methods}

\section{Materials}

All chemicals were from Sigma (Taufenkirchen, Germany) unless otherwise indicated.

\section{Source and collection of oocytes}

Bovine ovaries, collected with aseptic precautions from a local slaughter house, were transported to the laboratory in $0.9 \%$ physiological saline (at $20-25^{\circ} \mathrm{C}$ ) in a Thermos container within $2 \mathrm{~h}$ of slaughtering and were immediately washed after arrival in freshly made phosphate-buffered saline (PBS).

To collect the cumulus cell-oocyte complexes (COCs), the surfaces of the ovaries were slit open with a blade, according to the ovarian-slicing method (Eckert \& Niemann 1995) and washed with PBS solution $(100 \mathrm{ml}$ PBS per ovary). The flushing fluid so collected was sieved through a micro-pore sieve, with a pore size of $50 \mu \mathrm{m}$. The COCs were rinsed with PBS and collected in a Petri dish. The COCs were then picked up, sorted out and only COCs with compact layers of cumulus cells and evenly granulated ooplasm were selected for further investigations.

\section{Oocyte maturation and treatment of oocytes with the phosphatidylinositol analogue $\mathrm{SH} 6$}

The COCs collected were matured according to the method of Torner et al. (2001) in TCM 199, supplemented with $3 \mathrm{mg} / \mathrm{ml}$ bovine serum albumin (BSA), covered with a layer of mineral oil, at $38.5^{\circ} \mathrm{C}, 5 \% \mathrm{CO}_{2}$ and saturated humidity for different time intervals $(0,10$ and $24 \mathrm{~h}$ ). The IVM was performed in four-well dishes with 50 COCs in $500 \mu \mathrm{l}$ medium. To assess the influence of the phosphatidylinositol analogue SH6 (D-2,3-dideoxy-myo-inositol 1-[(R)-2-methoxy-3-(octadecyloxy)propyl hydrogen phosphate]; Alexis, Grünberg, Germany), the oocytes were matured in a maturation medium containing SH6 (final concentration of $10,25,50$ or $75 \mu \mathrm{M}$, prepared from a $50 \mathrm{mM}$ stock solution) dissolved in $\mathrm{H}_{2} \mathrm{O} . \mathrm{H}_{2} \mathrm{O}$ without $\mathrm{SH} 6$ was added to the control groups. After maturation, cumulus cells were removed by treatment with hyaluronidase $(2.5 \mathrm{mg} / \mathrm{ml})$ for $2 \mathrm{~min}$ and repeated pipetting. Then the oocytes were evaluated for the stage of meiosis by staining with aceto-orcein, or used for biochemical analysis. The analysis of samples was carried out in welldefined time periods (Torner et al. 2001) of 0,10 and $24 \mathrm{~h}$ of IVM (GV, M I and M II stages) respectively.

\section{Morphological evaluation}

In order to establish that the oocytes collected at the defined time points of maturation were actually at the 
aforementioned stages of maturation, some of them were randomly picked and fixed with acetic alcohol (acetic acid:ethanol $=1: 3$ ) and stained with the aceto-orcein dye to enable their classification into the stages of GV, GVBD, M I, anaphase I, telophase I and M II with extruded polar body (M II).

\section{One-dimensional (1D) gel electrophoresis (SDS-PAGE)}

To separate the proteins according to their apparent molecular masses, SDS-PAGE was performed in 10\% gels, according to Laemmli (1970). The ratio of acrylamide to bisacrylamide was 30:0.8 to analyse Akt or 29.7:0.3 to analyse MAPK.

After maturation, the oocytes were washed five times in protein-free PBS, lysed in $5 \mu \mathrm{l}$ double-concentrated SDS sample buffer, kept at $95^{\circ} \mathrm{C}$ for 2 min and immediately thereafter subjected to electrophoresis. Extracts of 50 oocytes per time point were analysed on the gels. Electrophoresis was performed from anode to cathode at $65 \mathrm{~mA}$ per gel, until bromophenol blue reached the bottom of the gel. For all 1D SDS-PAGE, the Mighty Small SE 250 system (Hoefer, Amersham Biosciences, Freiburg, Germany) was used.

\section{Western blotting and immunodetection}

The proteins separated by 1D gel electrophoresis (from 50 oocytes per time point) were transferred from the gels onto PVDF membranes (Millipore, Schwalbach, Germany) according to the method of Towbin \& Staehlin (1979) using a semidry electroblotting apparatus (PEQLab, Erlangen, Germany). The transfer was effected at $1 \mathrm{~mA} / \mathrm{cm}^{2}$ for $1 \mathrm{~h}$. To prevent non-specific binding, the free binding sites of the membranes were saturated with $5 \%$ dry milk in Tween-Tris-buffered saline (TTBS; $20 \mathrm{mM}$ Tris- $\mathrm{HCl}, \mathrm{pH}$ $7.4,137 \mathrm{mM} \mathrm{NaCl}$ and $0.1 \%$ Tween 20 ) for $1 \mathrm{~h}$ at room temperature. Thereafter, they were thoroughly washed three times for $10 \mathrm{~min}$ each time with $50 \mathrm{ml}$ TTBS. After every incubation the membranes were washed three times with TTBS. The membranes were probed with a polyclonal Akt antibody which recognizes the total Akt (Akt 1,2 and 3) or with two phospho-Akt (pAkt)-specific antibodies against epitopes containing Thr 308 or Ser 473 phosphorylated forms (NEB, Cell Signalling, Frankfurt, Germany). NIH/3T3 cell extracts were used as a positive control. As a negative control NIH/3T3 cells treated with phosphatidylinositol 3kinase inhibitor, 2-(4-morpholinyl)-8-phenyl-4H-1-benzopyran-4-one (LY294002; NEB) $(10 \mu \mathrm{M})$ were used; this had been previously shown to prevent Akt phosphorylation. The MAPK antibody which recognizes both forms, ERK 1 and ERK 2, was from Santa Cruz, Heidelberg, Germany.

In all cases, the antibody dilutions used for the primary was $1: 1000$. The incubation time was $16 \mathrm{~h}$ at $4{ }^{\circ} \mathrm{C}$. This was followed by the incubation of the blots with the secondary antibody (goat anti-rabbit horseradish peroxidase (HRP) labelled, diluted 1:3000, incubation $1.5 \mathrm{~h}$ at room temperature). The membranes were again washed three times for
10 min each time. Subsequently, the segregated proteins were visualized with the help of a chemiluminescence kit (ECL; Amersham Pharmacia Biotech, Freiburg, Germany) on X-ray films according to the manufacturer's instructions.

As a loading control and internal standard, the membranes were reprobed with an antibody against total actin (all isoforms from Sigma). For this purpose, after the chemiluminescent detection, the first antibody was stripped from the membrane by incubation in $100 \mathrm{mM}$ 2-mercaptoethanol, $2 \% \mathrm{SDS}$ and $62.5 \mathrm{mM}$ Tris $-\mathrm{HCl}, \mathrm{pH} 6.7$ for $10 \mathrm{~min}$ at $50^{\circ} \mathrm{C}$ under permanent agitation. After washing in TTBS, the membrane was incubated with the anti-actin antibody diluted 1:2000 in TTBS overnight at $4{ }^{\circ} \mathrm{C}$. The secondary anti-rabbit HRP-labelled antibody (1:4000) was incubated for $2 \mathrm{~h}$ at room temperature. Actin was detected by chemiluminescent reaction as described. In all cases, the optical density (OD) of the bands was measured by Scanalytics-1D-software (Scanalytics Inc., Fairfax, VA, USA). For the evaluation, the ratio of the OD of the protein concerned (Akt or pAkt) in relation to actin is presented in the form of bar charts.

\section{Kinase assays}

\section{Akt kinase assays}

To perform the Akt kinase assay with glycogen synthase kinase (GSK3) as an external ch substrate, the cell signalling kit from NEB was used. Ten oocytes per time point were lysed and Akt was immunoprecipitated using an immobilized monoclonal Akt antibody. The kinase assay was performed according to the manufacturer's instructions. After the assay, the lysates were separated by SDSPAGE according to Laemmli (1970) and transferred to PVDF membranes as described above. The GSK3 or phosphorylated GSK3 $\alpha$ at Ser 21 and GSK3 $\beta$ at Ser 9 was detected by a corresponding antibody (NEB) and visualized by chemiluminescent reaction on X-ray films as described above.

\section{Histone H 1 kinase assay}

The activity of histone $\mathrm{H} 1$ kinase reflecting maturationpromoting factor (Cdc2) kinase was measured in oocytes via the capacity to phosphorylate the external substratehistone $\mathrm{H} 1$ protein. At each time interval, ten oocytes per sample were collected. The histone $\mathrm{H} 1$ phosphorylation was measured as described by Motlik et al. (1996). Briefly, $5 \mu \mathrm{l}$ buffer A (40 mM MOPS, pH 7.2, $20 \mathrm{mM}$ para-nitrophenyl phosphate, $40 \mathrm{mM} \beta$-glycerophosphate, $10 \mathrm{mM}$ EGTA, $0.2 \mathrm{mM}$ EDTA, $2 \mathrm{mM}$ dithiothreitol, $0.2 \mathrm{mM} \mathrm{Na}_{3} \mathrm{VO}_{4}, 2 \mathrm{mM}$ benzamidine, $40 \mu \mathrm{g} / \mathrm{ml}$ leupeptin and $40 \mu \mathrm{g} / \mathrm{ml}$ aprotinin) was added to each sample. The tubes were briefly vortexed and centrifuged at $10000 \mathrm{~g}$ for $15 \mathrm{~s}$. The kinase reaction was initiated by the addition of $5 \mu$ l buffer B $(100 \mathrm{mM}$ MOPS, pH 7.2, $20 \mathrm{mM}$ para-nitrophenylphosphate, $40 \mathrm{mM}$ $\beta$-glycerophosphate, $20 \mathrm{mM} \mathrm{MgCl} 2,10 \mathrm{mM}$ EGTA, $0.2 \mathrm{mM}$ EDTA, $5 \mu \mathrm{M}$ cAMP-dependent protein kinase inhibitor, $2 \mathrm{mM}$ benzamidine, $40 \mu \mathrm{g} / \mathrm{ml}$ leupeptin, $40 \mu \mathrm{g} / \mathrm{ml}$ 
aprotinin, $600 \mu \mathrm{M}$ ATP and $2 \mathrm{mg}$ histone $\mathrm{H} 1 / \mathrm{ml}$ ) with $500 \mu \mathrm{Ci} / \mathrm{ml} \quad\left[{ }^{32} \mathrm{P}\right] \gamma$-ATP $(10 \mathrm{mCi} / \mathrm{ml}$; Amersham Pharmacia Biotech). The reaction was carried out for exactly $30 \mathrm{~min}$ at $30^{\circ} \mathrm{C}$ and stopped instantaneously by the addition of $10 \mu \mathrm{l}$ double-concentrated SDS-PAGE sample buffer and boiling for $3 \mathrm{~min}$. After electrophoresis on 15\% SDS-PAGE gel (Laemmli 1970), the gels were stained with Coomassie Blue R250, destained overnight, dried and autoradiographed.

\section{Statistical analysis}

In all cases, the data from three independent experiments were evaluated. The data of the Western blots, morphological analysis of oocytes during IVM and in response to SH6 treatment are expressed as means \pm S.D. of triplicate measurements. All data were analysed by one-way ANOVA (Sigma Stat, version 1.0; Jandel Corporation, San Rafael, CA, USA). If the effects of the main treatment were significant, a $t$-test was performed $(P<0.05)$.

\section{Results}

\section{Analysis of Akt abundance, phosphorylation and activity during IVM}

In Fig. 1, a typical profile of the evaluation of the chromatin configuration during IVM of bovine oocytes is presented. It can be seen that at $0 \mathrm{~h}$ of IVM approximately $90 \%$ of the oocytes persisted in the GV stage. After 10 or $24 \mathrm{~h}$ of IVM the majority of oocytes had reached M I and M II respectively. These observations are in accordance with results from Torner et al. (2001). For this purpose, oocytes derived at these time points were used for further biochemical investigations.

Western blot analysis of the extracts from oocytes from GV, M I and M II stages revealed that the protein kinase Akt, which has a theoretical molecular mass of $55 \mathrm{kDa}$, exhibited on SDS gels an apparent molecular mass of approximately $63 \mathrm{kDa}$ (this result is in accordance with data provided by the distributor of the antibody, Cell Signalling Technology). Furthermore, it can be seen that Akt is equally abundant during the time points investigated, as compared with actin as an internal standard (Fig. 2A, D and $\mathrm{H}$ ). This would indicate that the overall expression (sum of de novo synthesis and degradation) of this kinase

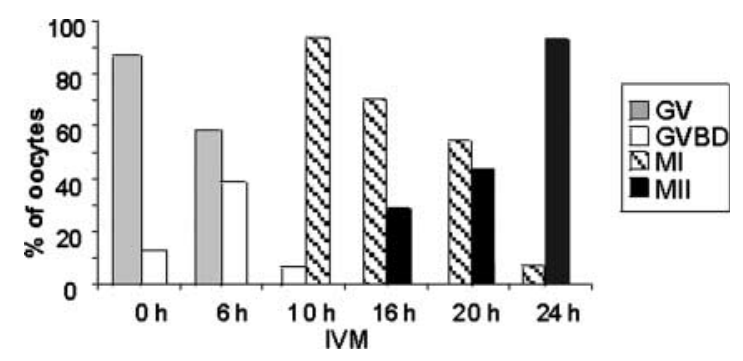

Figure 1 Morphological analysis of bovine oocytes in the course of IVM. Fifty oocytes per time point were stained with aceto-orcein and classified according to their chromatin configuration in GV, GVBD, M I and M II stages. remains constant during IVM. In contrast to this finding, the analysis of the phosphorylation of Akt at Thr 308 and Ser 473 resulted in enhanced signals after $10 \mathrm{~h}$ of IVM. At the time point $0 \mathrm{~h}$ and at $24 \mathrm{~h}$ of IVM, only low, basal levels of phosphorylation could be detected on both sides (Fig. 2B, $\mathrm{C}$ and $\mathrm{H}$ ). This observation indicated that Akt is activated around the M I stage in bovine oocytes. This interpretation was confirmed by performing an in vitro kinase assay with immunoprecipitated Akt and GSK3 as an external substrate. The results presented in Fig. 2E show that the precipitated Akt from oocyte extracts derived after $10 \mathrm{~h}$ of IVM has the highest ability to phosphorylate the external substrate. In Fig. 2F, the loading control of total GSK3 from the same blot is presented. The maturation process was confirmed by analysing the MAPK ERK 1 and ERK 2 by band shift assay on Western blots (Fig. 2G).

The evaluation of Akt abundance and phosphorylation from three independent experiments by the ratio of Akt or pAkt in relation to the loading control actin is presented in Fig. $2 \mathrm{H}$ in the form of bar charts.

A more detailed analysis of Akt activation is shown in Fig. $3 \mathrm{~A}$ and $\mathrm{B}$. The kinase assay presented revealed that already at the time of or directly after GVBD at 6 and $8 \mathrm{~h}$ of IVM, an enhanced phosphorylation of GSK3 could be observed as compared with the GV and M II stages.

Taken together, the results indicated that the protein kinase Akt becomes activated around the GVBD and M I stages and that this activation corresponds with the phosphorylation of this particular kinase. In the M II stage, only basal activity comparable with that found in the GV stage could be detected. It should be mentioned in this context that the antibody used did not discriminate between different isoforms of Akt. Therefore the activation profile reflects the sum of the activity of Akt 1, 2 and 3 potentially present in the oocytes.

\section{Influence of the phosphatidylinositol analogue SH6 on Akt phosphorylation at Thr 308 and Ser 473 during IVM}

As described before, Akt phosphorylation and activity was highest in M I-stage oocytes (10 h of IVM). To analyse the influence of $\mathrm{SH} 6$ on the maturational phosphorylation of this kinase, we cultivated oocytes for $10 \mathrm{~h}$ in the presence of different concentrations of SH6 (20-75 $\mu \mathrm{M})$. Our previous analysis revealed that concentrations lower than $20 \mu \mathrm{M}$ had no pronounced effect whereas concentrations higher than $75 \mu \mathrm{M}$ were cytotoxic (authors' unpublished results). For this reason these concentrations were not used in further experiments. The results obtained by Western blotting are presented in Fig. 4. It is apparent that SH6 had no significant effect on the abundance of Akt in comparison with the loading control actin (Fig. 4A, B and E). On the contrary, concentrations of 25, 50 and $75 \mu \mathrm{M} \mathrm{SH} 6$ significantly reduced the phosphorylation on both sides, Thr 308 and Ser 473 (Fig. 4C and D, lanes 2-4 and E), though this process was reversible. This reversibility was 

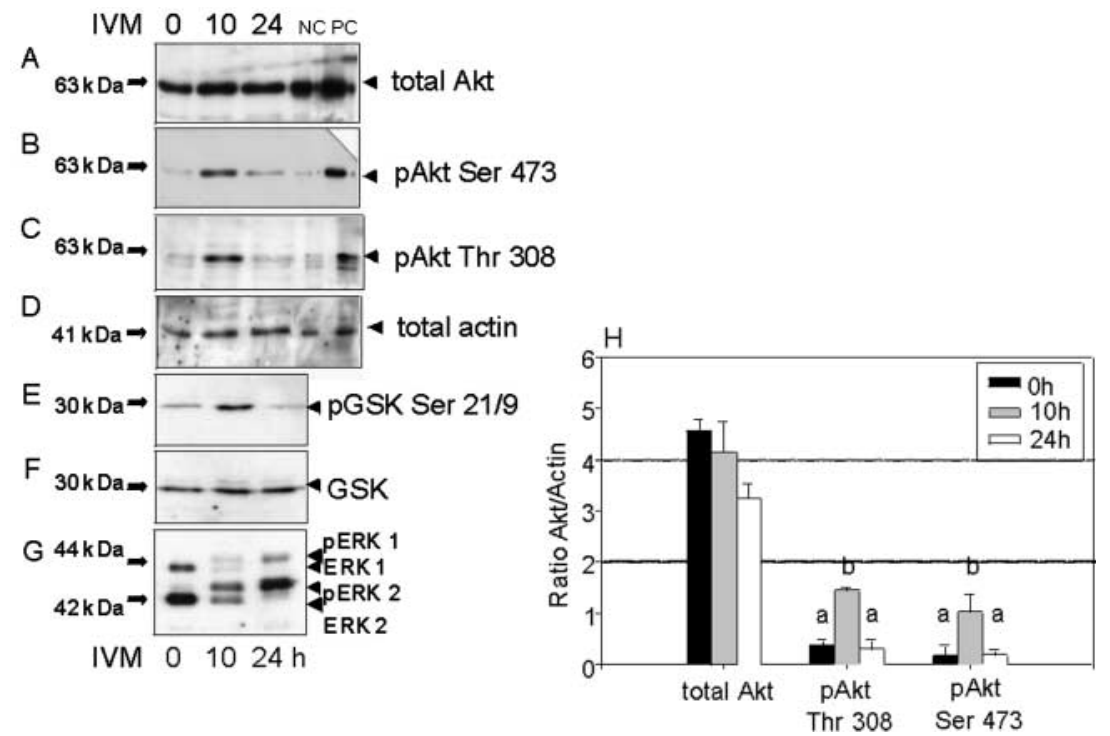

Figure 2 Abundance and activation of Akt during IVM. Fifty oocytes were matured to reach the GV, M I and M II stages respectively $(0,10$, 24 $\mathrm{h}$ IVM). Oocyte extracts were subjected to SDS-PAGE and analysed by Western blotting. In (A) the abundance of Akt was analysed with an antibody against total Akt. NIH/3T3 cell extracts served as a positive control (PC). Negative control (NC) was NIH/3T3 cells, treated with LY294002 $(10 \mu \mathrm{M})$ which was previously shown to prevent Akt phosphorylation. (B) and (C) show the phosphorylation of Akt during IVM at Ser 473 and Thr 308 respectively as obtained by the use of phosphospecific antibodies. (D) The blot from (A) was reprobed with an antibody against total actin as an internal standard. (E) Kinase assay with immunoprecipitated Akt and GSK3 as an external substrate. The enhanced phosphorylation of the substrate reflects enhanced Akt activity. (F) The same blot as shown in (E) was reprobed with an antibody against total GSK3 as a loading control. (G) The maturation process was confirmed by analysing the phosphorylation of MAPK in the supernatant of the Akt-immunoprecipitated oocyte lysates by band shift on Western blots. In all cases, representative blots of three independent experiments are shown. $(\mathrm{H})$ Evaluation of the abundance and phosphorylation state of Akt. The OD of bands generated by Akt and pAkt antibodies as depicted in (A-C) from three independent experiments was measured. The bar chart shows the values of total Akt or the phosphorylated forms of the kinase in relation to the internal standard total actin. Standard deviations are depicted. Bars with different superscripts are significantly different $(P<0.05)$.

demonstrated by the additional cultivation of the oocytes for $10 \mathrm{~h}$ without the inhibitor (Fig. 4C and D, lane 5).

\section{Morphological evaluation of SH6-treated oocytes}

An experiment was designed in which oocytes were maturated for $24 \mathrm{~h}$ in the presence of $\mathrm{SH} 6$. Under control conditions, oocytes reach the $M$ II phase in this time period; thus with this experiment, morphological alterations which were caused by impaired Akt activation could be analysed. The results from three independent experiments are presented in Fig. 5. Besides the controls (in lane 1, 24 IVM without SH6; in lane 6, 24 h IVM with

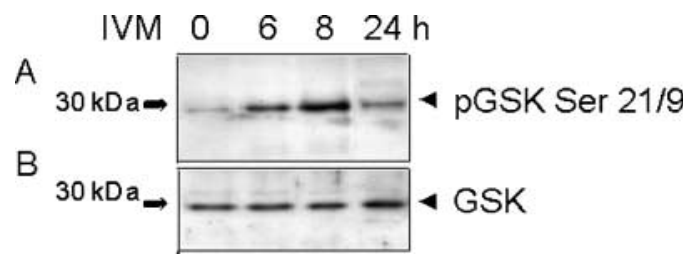

Figure 3 Analysis of Akt activity around the time of GVBD. Twenty oocytes per time point were matured for $0,6,8$ and $24 \mathrm{~h}$ to reach GV, GVBD and M II stages. Kinase assay was performed with immunoprecipitated Akt and GSK3 as an external substrate. The enhanced phosphorylation of the substrate reflects enhanced Akt activity. (B) The same blot as shown in (A) was reprobed with an antibody against total GSK3 as a loading control.
$50 \mu \mathrm{M} \mathrm{SH} 6$ followed by an additional $24 \mathrm{~h}$ of cultivation without the inhibitor), different concentrations of $\mathrm{SH} 6$ were applied (10, 25, 50 and $75 \mu \mathrm{M}$ in lanes 2-5). Fifty oocytes were evaluated in each group per experiment. The results showed that under control conditions $75-80 \%$ of the oocytes reached the M II stage after $24 \mathrm{~h}$ of IVM. The lowest concentration of SH6 $(10 \mu \mathrm{M})$ had no obvious effect on the progression of the maturation process. On the other hand, increasing concentrations of $\mathrm{SH} 6$ also increased the number of oocytes which persisted in M I even after $24 \mathrm{~h}$ of maturation (up to $60 \%$ compared with $20 \%$ in the control group). Thus, the results showed that the inhibition observed is far from being complete (see Discussion). The reason for this effect is not clear, but even longer cultivations (up to $30 \mathrm{~h}$ ) did not alter the values observed (data not shown). Obviously the concentration used had no cytotoxic effect nor did it induce apoptosis, since the inhibition was for the most part reversible (Fig. 3, lane 6).

\section{Evaluation of the side effects of SH6 on MAPK and MPF}

In order to exclude unspecific side effects of $\mathrm{SH} 6$ on MAPK and MPF activation during IVM, oocytes were matured for 10 or $24 \mathrm{~h}$ in the presence of different concentrations of this particular inhibitor. In Fig. 6A the phosphorylation of the MAPK ERK 2 analysed by band shift 

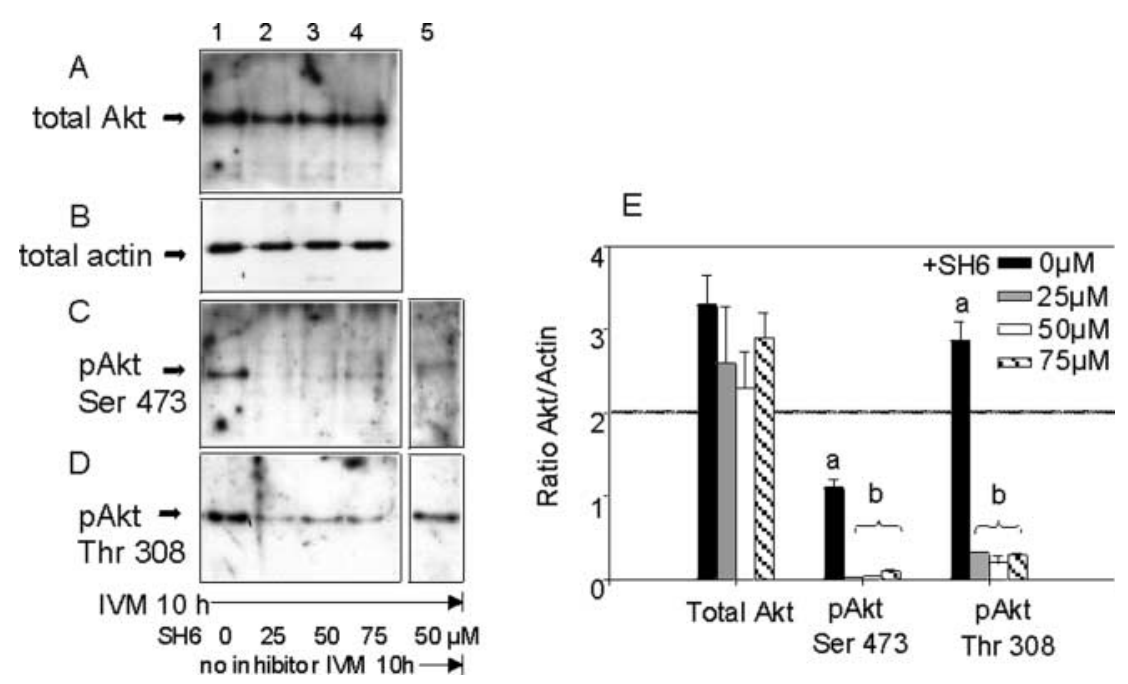

Figure 4 Inhibiton of Akt phosphorylation by treatment with SH6. Fifty oocytes per group were maturated for $10 \mathrm{~h}$ in the presence of different concentrations of SH6 $(0-75 \mu \mathrm{M}$; lanes 1-4) and analysed by Western blotting for the abundance and phosphorylation of Akt with the same antibodies as in Fig. 2A-C. In lane 5, oocytes were matured for $10 \mathrm{~h}$ in the presence of SH6 followed by an additional $10 \mathrm{~h}$ without inhibitor. (A) Analysis of the abundance of total Akt in the presence of SH6. (B) Analysis of the abundance of total actin as an internal standard, (C) and (D) analysis of the phosphorylation of Akt at Ser 473 and Thr 308 as described. Representative blots from three independent experiments are shown. (E) Evaluation of abundance and phosphorylation state of Akt in response to SH6 treatment. The OD of bands generated by Akt and pAkt antibodies as depicted in (A, C and D) from three independent experiments were measured. The bar chart shows the values of total Akt or the phosphorylated forms of the kinase in relation to the internal standard total actin. Standard deviations are depicted and bars with different superscripts are significantly different $(P<0.05)$.

assay on Western blots is depicted. It can be seen that ERK 2 was unphosphorylated at $0 \mathrm{~h}$ of IVM. At $10 \mathrm{~h}$ approximately half of the kinase was shifted to lower electrophoretic mobility even in the presence of different concentrations of $\mathrm{SH} 6$, indicating its partial phosphorylation, comparable with the extent obtained in the control group. Finally, ERK 2 was fully phosphorylated at $24 \mathrm{~h}$ IVM which was not influenced by $50 \mu \mathrm{M} \mathrm{SH} 6$.

Corresponding results were obtained by histone $\mathrm{H} 1$ kinase assay (Fig. 4B). The phosphorylation of the external substrate histone $\mathrm{H} 1$ reflects the activity of the MPF in the oocyte extract. These extracts were derived at the same maturation times as from the MAPK analysis. It can be seen that $\mathrm{SH} 6$ had apparently no influence on MPF activation.

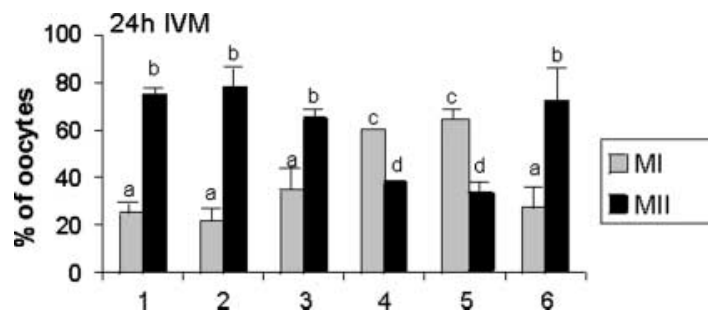

Figure 5 Morphological evaluation of Akt inhibition during IVM. Oocytes (50 per lane) were matured for $24 \mathrm{~h}$ in the presence of different concentrations of SH6 (lanes 1-5; 0, 10, 20, 50 and $75 \mu \mathrm{M} \mathrm{SH6}$ ). In lane 6, oocytes were matured for $24 \mathrm{~h}$ in the presence of $50 \mu \mathrm{M}$ $\mathrm{SH} 6$ followed by an additional $10 \mathrm{~h}$ of cultivation without the inhibitor. The oocytes were stained with aceto-orcein and classified according to their chromatin configuration. This experiment was performed three times and standard deviations are depicted. Bars with different superscripts are significantly different $(P<0.05)$.
Taken together, these results indicated that the inhibitory effect of SH6 was not caused by alterations of MAPK and/or MPF activation in the groups treated and must considered to be caused exclusively by Akt inhibition.

\section{Discussion}

In the present study we have investigated the abundance and phosphorylation state of the serine/threonine kinase Akt (which is also referred as PKB) during IVM of bovine oocytes. We used the phosphatidylinositol analogue SH6

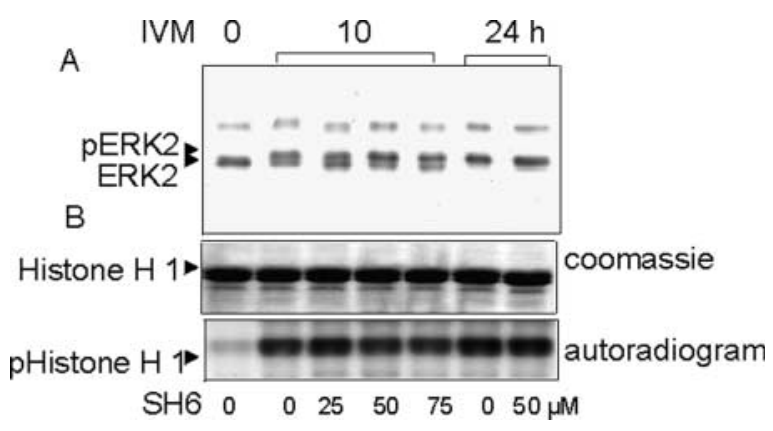

Figure 6 Influence of SH6 treatment during IVM on MAPK phosphorylation and MPF activation. Ten oocytes were matured for 0,10 or $24 \mathrm{~h}$ with the indicated concentrations of SH6. (A) Analysis of MAPK phosphorylation by band shift on Western blots. (B) The upper gel shows the external substrate histone $\mathrm{H} 1$ added to the oocyte extracts to perform the in vitro kinase assay (loading control). The gel was stained by Coomassie Blue. In the lower gel, the autoradiogram of the gel of the in vitro kinase assay reflecting the activity of MPF is depicted. The oocytes were cultivated in the presence of different concentrations of $\mathrm{SH} 6$ for different times as indicated. 
as a tool to investigate Akt function during meiotic maturation of bovine oocytes.

The phosphatidylinositol analogue $\mathrm{SH} 6$ is a novel inhibitor which was shown to prevent Akt activation specifically in somatic cells (Kozikowski et al. 2003, Chrysis et al. 2005) but it had not been used before to investigate the maturation processes of oocytes. One advantage of this particular inhibitor is its potential selectivity for Akt. In contrast, other substances such as wortmannin or LY294002 which have been shown to influence the Aktdependent signalling cascade, were shown to act on the upstream phosphatidylinositol-3-OH kinase and therefore influence the activity of the PDK1 (Vlahos et al. 1994, Burgering \& Coffer 1995, Franke et al. 1995). Therefore unspecific side effects of wortmannin or LY294002 on, for instance, p70S6K and protein kinase C have to be considered (Kozikowski et al. 2003, Scheid \& Woodgett 2003, Tang \& McLeod 2004).

Like MAPK (Kubelka et al. 2000, Tomek et al. 2002a, present study) Akt could be detected during the whole maturation process without any significant alteration in abundance at the GV, M I and M II stages. It should be mentioned here that this result is not in agreement with observations made by Vigneron et al. (2004b). These authors reported no or only very low abundance of Akt in the GV stage (time 0) of bovine oocytes. This discrepancy is probably the result of the use of different antibodies. The antibodies used in our study (one against total Akt, and two against pAkt Ser 308 or Thr 473) recognize all three isoforms of Akt, which all show an apparent molecular mass of $63 \mathrm{kDa}$ on SDS gels. In the study cited, no information about the specifity of the antibody used is given.

In contrast to MAPK, which becomes activated at the time of GVBD and shows a maximum of activity in the M II stage (Kubelka et al. 2000), the kinase Akt is mainly active in the M I stage, as is confirmed by the analysis of the phosphorylation pattern and by in vitro kinase assay. When MPF and/or MAPK activation is blocked, e.g. by the CDK inhibitor buryrolactone I or by roscovitine, oocytes persist in the GV stage (e.g. Kubelka et al. 2000, Lonergan et al. 2003, Vigneron et al. 2004a). On the other hand, the present study revealed that the majority of the oocytes persist in the M I stage when they were matured in the presence of SH6. Our observations have indicated that this is an effect exclusively of Akt inhibition, since no side effects of $\mathrm{SH} 6$ on MAPK or MPF activation could be observed.

Although we obtained a strong inhibition of Akt activation during IVM by SH6, surprisingly a considerable number of oocytes reached the M II stage (approximately $40 \%$ at a concentration of $50 \mu \mathrm{M} \mathrm{SH} 6$ ). The reason for this observation is not clear at the moment. The most straightforward explanation for this effect would be to assume that a sufficient portion of activated Akt is already present in several GV-stage oocytes which allows them to overcome the inhibitory effect of SH6. But at the moment we do not have any convincing direct proof of this hypothesis.
Detailed analysis of somatic cells has revealed that Akt is involved in the regulation of cap-dependent translation (Sonenberg \& Gringas 1998, Gingras et al. 2001). This function is mediated by the ability of the Akt signalling cascade to phosphorylate (and inactivate) a specific inhibitor of the function of the mRNA-cap binding protein elF4E, namely its repressor protein $4 \mathrm{E}$ BP1. As recently revealed by our group, elF4E subsequently becomes phosphorylated during IVM (which should result in stimulation of translation) under the influence of MAPK; there is also a concurrent presence of 4E-BP1 in bovine oocytes (Tomek et al. 2002a, Smiljakovic et al. 2003). Furthermore, we have shown that protein synthesis during the IVM of bovine oocytes is at its maximum at the time of GVBD and during the M I stage (Tomek et al. 2002a,b) which are precisely the two stages of maturation in which Akt is active. Therefore, an attractive hypothesis concerning a particular Akt function during meiotic maturation of bovine oocytes would be to assume that Akt is involved in balancing protein synthesis by phosphorylating 4E-BP1. An inhibition of this regulatory process, for example by SH6, would result in M I arrest. We will try to verify this hypothesis in further experiments by investigating the effects of SH6 treatment on 4E-BP1 phosphorylation and on translation rates during the IVM of bovine oocytes.

\section{Acknowledgements}

We wish to thank Gesine Krüger for expert technical assistance and Marijana Max for her help in the cultivation of oocytes. This work was supported by the Deutsche Forschungsgemeinschaft (DFG), To 178/2-2. T S is a fellow of the German Academic Exchange Service (DAAD). The support is gratefully acknowledged. The authors declare that there is no conflict of interest that would prejudice the impartiality of this scientific work.

\section{References}

Burgering BM \& Coffer P 1995 Protein kinase B (c-Akt) in phosphatidylinositol-3-OH kinase signal transduction. Nature 17 553-554.

Chian RC, Chung J, Niwa K, Sirard MA, Downey BR \& Tan SL 2003 Reversible changes in protein phosphorylation during germinal vesicle breakdown and pronuclear formation in bovine oocytes in vitro. Zygote 11 119-129.

Chrysis D, Zaman F, Chagin AS, Takigawa M \& Savendahl L 2005 Dexamethasone induces apoptosis in proliferative chondrocytes through activation of caspases and suppression of the Akt-phosphatidylinositol 3'-kinase signaling pathway. Endocrinology 146 1391-1397.

Claude R, Isabelle H, Serge MG, Dominic G \& Marc-Andre S 2002 Quantification of cyclin B1 and p34 ${ }^{\text {Cdc2 }}$ kinase in bovine-cumulus oocyte complexes and expression mapping of genes involved in the cell cycle by complementary DNA microarrays. Biology of Reproduction 67 1456-1464.

Dorée M \& Hunt T 2002 From cdc2 do cdk1: when did the cell cycle kinase join its partner? Journal of Cell Science 115 2461-2464. 
Eckert J \& Niemann H 1995 In vitro maturation, fertilization and culture to blastocysts of bovine oocytes in protein-free media. Theriogenology 43 1211-1225.

Evans T, Rosenthal ET, Youngblom J, Distel D \& Hunt T 1983 Cyclin: a protein specified by maternal mRNA that is destroyed at each cleavage division. Cell 33 389-396.

Franke TF, Yang SI, Chan TO, Datta K, Kazlauskas A, Morrison DK, Kaplan DR \& Tsichlis PN 1995 The protein kinase encoded by the Akt proto-oncogene is a target of the PDGF-activated phosphatidylinositol 3-kinase. Cell 81 727-736.

Gerhard JC, Wu M \& Kirschner M 1984 Cell cycle dynamics of an M-phase specific cytoplasmic factor in Xenopus laevis oocytes and eggs. Journal of Cell Biology 98 1247-1255.

Gingras AC, Raught B, Gygi SP, Niedzwiecka A, Miron M, Burley SK, Polakiewicz RD, Wyslouch-Cieszynska A, Aebersold R \& Sonenberg $\mathbf{N} 2001$ Hierarchical phosphorylation of the translation inhibitor 4E-BP1. Genes and Development 15 2852-2864.

Hoshino Y, Yokoo M, Yoshida N, Sasada H, Matsumoto H \& Sato E 2004 Phosphatidylinositol 3-kinase and Akt participate in the FSHinduced meiotic maturation of mouse oocytes. Molecular Reproduction and Development 69 77-86.

Jessus C \& Beach D 1992 Oscillation of MPF is accompanied by periodic association between cdc25 and cdc2-cyclin B. Cell 68 $323-332$

Jones KT 2004 Turning it on and off: M-phase promoting factor during meiotic maturation and fertilization. Molecular Human Reproduction $101-5$.

Kishimoto T 2003 Cell-cycle control during meiotic maturation. Current Opinion in Cell Biology 15 654-663.

Kozikowski AP, Sun H, Brognard J \& Dennis PA 2003 Novel PI analogues selectively block activation of the pro-survival serine/threonine kinase Akt. Journal of the American Chemical Society 125 1144-1145.

Kubelka M, Motlik J, Schultz RM \& Pavlok A 2000 Butyrolactone I reversibly inhibits meiotic maturation of bovine oocytes, without influencing chromosome condensation activity. Biology of Reproduction 62 292-302.

Laemmli UK 1970 Cleavage of structural proteins during the assembly of the head of bacteriophage T4. Nature 227 680-685.

Lonergan P, Faerge I, Hyttel PM, Boland M \& Fair T 2003 Ultrastructural modifications in bovine oocytes maintained in meiotic arrest in vitro using roscovitine or butyrolactone. Molecular Reproduction and Development 64 369-378.

Lui L \& Yang X 1999 Interplay of maturation-promoting factor and mitogen-activated protein kinase inactivation during metaphase-tointerphase transition of activated bovine oocytes. Biology of Reproduction 61 1-7.

Massagué J 2004 G1 cell-cycle control and cancer. Nature 423 298-306.

Moor RM, Mattioli M, Ding J \& Nagai T 1990 Maturation of pig oocytes in vivo and in vitro. Journal of Reproduction and Fertility 40 Suppl 197-210.

Motlik J, Sutovsky P, Kalous J, Kubelka M, Moos J \& Schultz RM 1996 Co-culture with pig membrana granulosa cells modulates the activity of cdc2 and MAP kinase in maturing cattle oocytes. Zygote 4 247-256.

Nebreda A \& Hunt T 1993 The c-mos proto-oncogene protein kinase turns on and maintains the activity of MAP kinase, but not MPF, in cell-free extracts of Xenopus oocytes and eggs. EMBO Journal 12 1979-1986.

Okumura E, Fukuhara T, Yoshida H, Hanada S, Kozutsumi R, Mori M, Tachibana K \& Kishimoto T 2002 Akt inhibits Myt1 in the signalling pathway that leads to meiotic G2/M-phase transition. Nature Cell Biology 4 111-116.

Sarbassov DD, Guertin DA, Ali SM \& Sabatini DM 2005 Phosphorylation and regulation of $\mathrm{Akt} / \mathrm{PKB}$ by the rictor-mTOR complex. Science 307 1098-1101.

Scheid MP \& Woodgett PA 2003 Unravelling the activation mechanisms of protein kinase B/Akt. FEBS Letters 546 108-112.
Scheid MP, Marignani PA \& Woodgett JR 2002 Multiple phosphoinositide 3-kinase dependent steps in activation of protein kinase B. Molecular and Cellular Biology 22 6247-6260.

Shibuya EK \& Ruderman JV 1993 Mos induces the in vitro activation of mitogen-activated protein kinases in lysates of frog oocytes and mammalian somatic cells. Molecular Biology of the Cell 4 781-790.

Sirard MA, Dufort I, Coenen K, Tremblay K, Massicotte L \& Robert C 2003 The use of genomics and proteomics to understand oocyte and early embryo functions in farm animals. Reproduction 61 Suppl 117-129.

Smiljakovic T, Melo Sterza F, Kubelka M, Vohnikova Z \& Tomek W 2003 Aspects of cytoplasmic maturation of bovine oocytes: interplay between MAPK, mRNA cap binding complex and cytoplasmic mRNA metabolism in regulation of translation. Biotechnology in Animal Husbandry 19 1-8.

Sonneburg N \& Gringas AC 1998 The mRNA 5'-cap binding protein elF4E and control of cell growth. Current Opinion in Cell Biology $10268-275$.

Tang Y \& McLeod M 2004 In vivo activation of protein kinase A in Schizosaccharomyces pombe requires threonine phosphorylation at its activation loop and is dependent on PDK1. Genetics $\mathbf{1 6 8}$ $1843-1853$.

Tian XC, Lonergan P, Jeong BS, Evans AC \& Yang X 2002 Association of MPF, MAPK, and nuclear progression dynamics during activation of young and aged bovine oocytes. Molecular Reproduction and Development 62 132-138.

Tomek W, Sterza Melo FA, Kubelka M, Wollenhaupt K, Torner H, Anger M \& Kanitz W 2002a Regulation of translation during in vitro maturation of bovine oocytes: the role of MAP kinase, elF4E (Cap binding protein) phosphorylation and elF4E-BP1. Biology of Reproduction 66 1274-1282.

Tomek W, Torner H \& Kanitz W 2002b Comparative analysis of protein synthesis, transcription and cytoplasmic polyadenylation of mRNA during maturation of bovine oocytes in vitro. Reproduction of Domestic Animals 37 86-91.

Torner H, Kubelka M, Heleil B, Tomek W, Alm H, Kuzmina T \& Guiard V 2001 Dynamics of meiosis and protein kinase activities in bovine oocytes correlated to prolactin treatment and follicle size. Theriogenology 55 885-899.

Towbin H \& Staehlin GJ 1979 Electrophoretic transfer of proteins from acrylamide gels to nitrocellulose sheets. PNAS 76 4350-4354.

Troussard AA, Mawji NM, Ong C, Mui A, St-Arnaud R \& Dedhar S 2003 Conditional knock-out of integrin-linked kinase demonstrates an essential role in protein kinase B/Akt activation. Journal of Biological Chemistry $27822374-22378$.

Vigneron C, Perreau C, Dalbies-Tran R, Joly C, Humblot P, Uzbekova S \& Mermillod P 2004a Protein synthesis and mRNA storage in cattle oocytes maintained under meiotic block by roscovitine inhibition of MPF activity. Molecular Reproduction and Development 69 457-465.

Vigneron C, Perreau C, Dupont J, Uzbekova S, Prigent C \& Mermillod P 2004b Several signaling pathways are involved in the control of cattle oocyte maturation. Molecular Reproduction and Development $69466-474$.

Vlahos CJ, Matter WF, Hui KY \& Brown RF 1994 A specific inhibitor of phosphatidylinositol 3-kinase, 2-(4-morpholinyl)-8-phenyl-4H-1benzopyran-4-one (LY294002). Journal of Biological Chemistry $2695241-5248$.

Ye J, Flint APF, Luck MR \& Campell KHS 2003 Independent activation of MAP kinase and MPF during the initiation of meiotic maturation in pig oocytes. Reproduction 125 645-656.

Received 11 April 2005

First decision 23 May 2005

Revised manuscript received 21 June 2005

Accepted 27 June 2005 